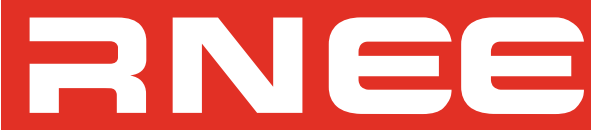

Revista Nicolaita de Estudios Económicos

Nueva Época

Instituto de Investigaciones Económicas y Empresariales
Universidad Michoacana de San Nicolás de Hidalgo

Volumen XVI, Número 2, Julio - Diciembre 2021, pp. 73 - 88

ISSN (Print): 1870 - 5464 ISSN (On-line): 2007 - 9877

DOI: $10.33110 /$ rnee.v16i2.330

(Recibido: 26/octubre/2021; Aceptado: 16/diciembre/2021)

\title{
Estructura económica e incidencia de pobreza en las regiones de México
}

\section{Economic structure and incidence of poverty in the regions of Mexico}

\author{
Carlos Padilla Morán a * \\ Joaquín Bracamontes Nevárez ${ }^{\mathrm{b}}$
}

\section{Resumen}

En este trabajo el objetivo es analizar si la estructura económica y los cambios que esta experimenta se correlacionan e inciden en la pobreza durante el periodo 2008-2018. Para ello se estima la participación sectorial, el efecto intrasectorial (EI) y el efecto de reasignación (ER) del empleo hacia sectores de alta productividad en las cuatro regiones que conforman el país: norte, tradicional centro y sur-sureste, delimitadas por entidades federativas que se caracterizan por su cercanía geográfica e intensidad migratoria. La participación sectorial en el empleo y en el PIB sugieren que la estructura económica tiende a terciarizarse en las cuatro regiones y a nivel nacional. En el país el aumento en la productividad se atribuye principalmente al ER, igual sucede en la región norte, pero en la región tradicional cuatro quintas partes del incremento en la productividad se debe al EI y el resto al ER, en la región centro ambos efectos guardan el mismo peso y, en la sur-sureste la reducción en la productividad se debe principalmente al EI y en menor medida al ER; sin embargo, las actividades a las que se reubica el empleo en todas las regiones son en su mayoría actividades de servicios y no industriales. Finalmente, se encontró que la reducción de la pobreza se explica por el crecimiento económico, la participación de los sectores secundario y terciario de la economía, pero no por el efecto de reasignación lo cual denota la inexistencia de un cambio estructural en las economías regionales y nacional.

Palabras clave: estructura económica, cambio estructural, productividad, pobreza.

\footnotetext{
a Profesor en la Universidad Tecmilenio. Dirección: Blvd. Juan Bautista Escalante \# 46, Recinto Alto Valle, 83106 Hermosillo, Sonora, México. Tel: +52 66218830 05. Email: carlos_padilla@tecmilenio.mx

b Profesor-Investigador en el Departamento de Economía Regional del Centro de Investigación en Alimentación y Desarrollo (CIAD A.C), Miembro del Sistema Nacional de Investigadores Nivel I, Tel: + 52 (662) 28924 00, Ext. 106. Email: joaco@ciad.mx. Dirección: Carretera a La Victoria km 0.6 C.P. 83304, Hermosillo, Sonora, México.

El trabajo deriva de la tesis de Maestría en Desarrollo Regional realizada por el primer autor con asesoría del coautor. Se agradece al Conacyt por el apoyo económico para cursar el posgrado en el CIAD

* Autor de correspondencia
} 


\section{Introducción}

En la actualidad, la pobreza es uno de los principales problemas para lograr el desarrollo a escala global (CEPAL, 2016, 2007). En este sentido, durante las últimas décadas, varios países han logrado notables resultados en la reducción de la pobreza, destacando China e India en el continente asiático y Brasil en América Latina (BM, 2018). Estos países transitaron de especializarse en materias primas a estructuras donde la actividad industrial tiene un peso cada vez más significativo. Este proceso está vinculado a la reducción de la pobreza (ONUDI, 2012).

No obstante, la pobreza sigue representando un problema considerable para países desarrollados y países en desarrollo, principalmente aquellos que orientaron su política económica en apego al esquema neoliberal. Entendido el neoliberalismo cómo la teoría o paradigma que sustenta las prácticas políticoeconómicas que sostienen que la mejor forma de promover el bienestar consiste en no restringir el libre desarrollo de las capacidades y libertades empresariales del individuo, con esto el rol del Estado es preservar el marco institucional caracterizado por fuertes derechos de propiedad privada, mercados libres y libre comercio (Harvey, 2005).

En el marco del neoliberalismo y en términos de la estructura económica, en México la participación en el PIB del sector terciario o de servicios -considerado de baja productividad- tendencialmente se ha incrementado al pasar de tener una participación relativa del 58.97\% en el PIB nacional del año 2000 a $65.63 \%$ en el año $2017^{1}$; mientras que, la participación disminuye en las manufacturas de 17.77\% en 2003 a 16.63\% en $2017^{2}$.

Las medidas de política económica que se ha adoptado durante las últimas décadas para el desarrollo nacional han dado como resultado que más de una tercera parte de la población se encuentra en pobreza por ingresos (CONEVAL, 2019). Esto se acentúa por el incremento en los precios que no es acompañado con el aumento de los salarios reales, además de la fuga de capitales provocada por las crisis económicas recurrentes dando como resultado la tercerización de la economía (Carrillo y Cadena, 2019), el incremento de la informalidad laboral, así como de la desigualdad y la pobreza regional (Camberos y Bracamontes, 2015).

El objetivo principal en este estudio es conocer la estructura económica de las diferentes regiones en México para indagar si el incremento en la productividad deriva de la relocalización del empleo o del incremento natural de esta y, a la vez, determinar si ello se correlaciona con los niveles de pobreza durante el período 20082018. Además de lo anterior, identificar los factores que determinan la pobreza en las regiones y el país.

El trabajo está compuesto por la introducción y otras cinco partes más. La segunda parte presenta una breve revisión del enfoque estructuralista y su relación con la pobreza, así como revisión de literatura con evidencia empírica. Luego se explica la metodología y datos utilizados. En la cuarta parte se analiza la estructura económica, el efecto intrasectorial (EI), el efecto de reasignación (ER) y la situación de pobreza en las regiones y el país; mientras que, la quinta parte da cuenta de los factores determinantes de la pobreza y, finalmente, se añaden las conclusiones del estudio.

\footnotetext{
1 Cálculos propios con datos de INEGI (2017, Cuentas Nacionales, PIB real a pesos del 2013).

2 Ibidem.
} 


\section{El enfoque estructuralista y la pobreza}

Para los pioneros del estructuralismo la estructura económica está fuertemente vinculada con las condiciones de vida de la población, ya que las diferencias estructurales entre países desarrollados y no desarrollados fundamentan los contrastes socioeconómicos de un tipo de país con respecto al otro (Dutt, 2019). El concepto de estructura económica alude a la clasificación sectorial propuesta por Fischer (1935 y 1939), a saber: el sector primario que comprende las actividades agrícolas, el sector secundario integrado por actividades industriales y mineras. Por último, el sector terciario, que incluye las actividades dedicadas a los servicios (Moncayo, 2008).

En este sentido, el enfoque estructuralista advierte que la estructura primario-exportadora, de servicios y poco industrializada explica en cierta medida las condiciones de atraso en cuanto a las capacidades de consumo y los niveles de pobreza en Latinoamérica y en el resto de los países no desarrollados. Esto, debido a la pérdida histórica del poder de compra de las materias primas con respecto a los productos industrializados en el mercado internacional (Prebisch, 1949, 1967; Furtado, 1961; Pinto, 1970; Cardoso y Faletto, 1979).

Para Moncayo (2008), el proceso que conlleva al crecimiento económico da lugar al cambio en la estructura económica, lo cual se constata a partir de la relocalización de la fuerza de trabajo alterando la ocupación relativa de un sector o rama hacia otros o a partir del cambio en la proporción que representa el PIB sectorial (y sus ramas) en la producción total; sin embargo, no todo cambio en la estructura económica implica un cambio estructural.

La tradición estructuralista latinoamericana define el cambio estructural como aquel que induce mejoría socioeconómica a partir del desarrollo de actividades industriales y tecnológicas, lo que significa una mayor productividad y crecimiento sostenido, mejorando así las condiciones de inserción en el mercado internacional. Esto implica un cambio de carácter virtuoso que aumenta la productividad general dando paso a una mejoría sustancial en las condiciones de vida de la población, lo que mitiga los problemas socioeconómicos en los países no desarrollados (CEPAL, 2012; Yoguel y Barletta, 2017).

En las últimas décadas, al seno de la CEPAL -como el estructuralismo clásico- surge el enfoque neoestructuralista, para el cual no todos los sectores tienen igual potencial para inducir aumentos en la productividad, encadenamientos productivos, empleos de alta remuneración o atender los problemas socioeconómicos (Cimoli et al., 2005). Es una crítica al estructuralismo clásico que no considera el peso del Estado y las instituciones para alcanzar el bienestar mediante mecanismos como las transferencias de ingresos, impuestos progresivos, así como su efecto redistributivo para reducir la desigualdad y la pobreza (Dutt, 2019).

De tal manera, la renovada perspectiva estructuralista asume que el Estado es un actor relevante en el impulso del desarrollo y en la promoción del cambio en la estructura económica para reducir las brechas en la productividad y los niveles de vida entre las naciones (Storm, 2015; Dutt, 2019). Además, el cambio estructural va más allá de la relocalización de la producción hacia el sector industrial, pues incluye las actividades intensivasdifusoras de conocimiento y con alta elasticidad-ingreso de la demanda de las exportaciones, en contraposición con las que lo son en recurso naturales o en trabajo (Cimoli et al. 2005; Cimoli et al. 2010; CEPAL, 2012).

Esto es, se trata de las actividades que hacen mayor uso de la tecnología, contrario a las que son intensivas en mano de obra o aquellas intensivas en recursos naturales como la minería (CEPAL, 2012). Por ello se sugiere el cambio estructural como el cambio en el patrón de especialización, en consideración a la forma en que la composición de los sectores se relaciona con el cambio tecnológico (Katz, 2000; Ocampo, 2005). 
Por lo tanto, para comprender el vínculo entre estructura económica, cambio estructural y bienestar es importante señalar que el dinamismo económico que transforme la estructura productiva es complementario a la igualdad social. De esta manera, el cambio estructural se entiende como el logro de una sinergia entre ambos en una visión integrada del desarrollo que alcance el crecimiento económico y los incrementos de la productividad, considerando la inclusión social y la sustentabilidad ambiental (Cimoli et al., 2005; CEPAL, 2012).

En cuanto a la relación entre la estructura económica y las condiciones de pobreza, la evidencia empírica muestra que los países que están atravesando procesos de industrialización y que orientan su fuerza laboral en actividades de esta naturaleza son los que históricamente registran la mayor productividad y han visto una disminución en sus niveles de pobreza, por lo que se constata un alto grado de causalidad. Este proceso se conoce como cambio estructural (Cimoli et al.2005; Lavopa, 2012; ONUDI 2012 y Berthélemy, 2018).

Sobre la relación entre la estructura económica y las condiciones de pobreza, la evidencia empírica muestra que los países que atraviesan por procesos de industrialización y que dirigen la fuerza de trabajo en actividades de esta índole son las que históricamente registran la mayor productividad y han visto disminuir sus niveles de pobreza encontrando alto grado de causalidad. A este proceso se le conoce como cambio estructural (Cimoli et al. 2005; Lavopa, 2012; ONUDI 2012; Berthélemy, 2018; Diao, McMillan y Rodrik, 2019).

También es posible apreciar que el aumento de la participación en las manufacturas fue acompañado con una reducción considerable de las condiciones de pobreza en Brasil (ONUDI, 2012; Lavinas y Somoes, 2016), Corea del Sur (Lanzarotti, 1991), China (ONUDI, 2012), África (Berthélemy, 2018) y Kazajistán (Verme, 2010); sin embargo, en Rusia (ONUDI, 2012), se redujo la pobreza y disminuyó la participación en el sector secundario; mientras que en Bangladesh resulta perjudicial en este sentido (Osmani, 1990).

Por otra parte, en el caso de Colombia (García, 2002), el Salvador (Segovia, 1998) y el continente africano (Berthélemy, 2018) un cambio en la estructura hacia el sector servicios no es acompañado con una considerable reducción de la pobreza, pero en los casos de la India (Aggarwal, 2012) o el Perú (Tello, 2015) cuando el empleo se dirige del sector agrícola a actividades de servicios tiene repercusiones positivas y considerables para reducir la pobreza, lo cual es atribuido a la productividad de los empleos a los que se va la fuerza de trabajo y los salarios que estos ofrecen.

El ejemplo de Perú, Tello (2015) muestra que al acrecentarse la participación del sector formal la pobreza se redujo, aunque también se advierte la relevancia de otros factores que participan en la reducción de la pobreza como el aumento del ingreso de los más pobres (Verme, 2010), el fortalecimiento del mercado interno (Lanzarotti, 1991) y las condiciones iniciales de los salarios como señala Berthélemy (2018). Este último autor además señala que en su estudio el crecimiento económico es función de la pobreza y no a la inversa como suele sugerirse para algunos países de África.

En México, el primer precedente sobre un análisis sobre el cambio en la estructura económica lo presenta Keesing (1969), el cual señala que del periodo de 1895 a 1950 cuando el país comienza a industrializarse, el autor compara la participación de la fuerza de trabajo en los sectores mediante tres censos en ese periodo (1895, 1930 y 1950). Encuentra que la agricultura pasa de representar $66.5 \%$ de la población ocupada a $58.32 \%$ y las actividades manufactureras de $11.51 \%$ a $12.62 \%$ relativamente mucho más bajo que las naciones industrializadas, por lo que indica que México debería enfocarse en la reasignación de la fuerza laboral hacia sectores no agrícolas, caracterizados por tener mayor productividad y ser mejor remunerados. 
Más recientemente, refiriéndose a la implementación de políticas neoliberales, Morton (2003), señala que México atraviesa "revolución pasiva" a partir del surgimiento del neoliberalismo durante el periodo de cambio estructural desde la década de 1970 como resultado de la reorganización del capitalismo a partir de la crisis del estado, el autor concluye que la transición del modelo sustitución de importaciones como forma de acumulación al neoliberalismo en México comienza en la década de 1970 como resultado de la naturaleza del capitalismo a nivel global y el papel que desarrollaría el país en el mercado internacional.

Respecto a las consecuencias en la estructura económica del modelo neoliberal, Capdevielle (2005) señala que en la actualidad, el principal valor agregado local que se incorpora al producto global en México es el correspondiente al trabajo empleado; las remuneraciones medias de los trabajadores de la industria maquiladora de exportación, si bien son superiores a las del conjunto de la economía, son más bajas que las que prevalecen en las industrias manufactureras; las empresas, pueden hacer uso de tecnología de punta sin que esto vaya acompañado del pago de remuneraciones más altas, esto es debido al escaso valor agregado en el proceso de producción y, asimismo, al uso limitado de insumos nacionales, ya que esta tecnología agrega valor en la cadena donde es producida y no donde es utilizada; por lo que concluye la heterogeneidad estructural de la economía mexicana se origina en la imposibilidad de crear capacidades productivas y tecnológicas.

Por otra parte, Adair (2006), en su trabajo examina la relación entre la desigualdad de ingresos y el crecimiento económico a partir del cambio en la estructura económica durante el periodo de liberación, el autor aplica análisis de correlación entre el crecimiento económico medido por el crecimiento del PIB y la desigualdad utilizando un índice de Gini calculado con los empleados del sector manufacturero. En el periodo de 1981 a 2003, el aumento en la desigualdad no se correlaciona con crecimiento económico, además el coeficiente no es significativo.

Respecto a la disminución de la pobreza explicado por el crecimiento económico, Campos y Monroy (2016) analizan esta relación para los estados de la república en el periodo de 2000-2014, aplicando método de regresión encuentran que no se observa una relación sistemática entre crecimiento y variaciones de pobreza; sin embargo, esta relación presenta un coeficiente mayor a 1 en estados con mejores condiciones de acceso a servicios básicos, mayor salario inicial, mayor grado de formalidad y con empleo especializado en manufacturas(Baja California, Baja California Sur, Chihuahua, Distrito Federal, Guanajuato, Jalisco, Nuevo León, Querétaro y Tamaulipas).

Otro estudio respecto a esta relación la ofrecen Iniguez-Montiel y Kurosaki (2015), en este trabajo los autores examina la efectividad del crecimiento económico y la redistribución reduciendo la pobreza en México durante el periodo de 1992 a 2012, utilizan método de descomposición y de regresión lineal para observar el cambio en la pobreza dividiendo los componentes a los que se debe y que tanto lo explican: el crecimiento económico, la distribución del ingreso y la inflación heterogénea calculada con la canasta de los pobres(ya que es superior a la media nacional). Los resultados muestran que la reducción de la pobreza se explica en gran medida por la distribución del ingreso.

Si bien los dos trabajos anteriores tratan centralmente la relación entre el crecimiento económico y la disminución en la pobreza. Los resultados dan soporte a que el crecimiento económico no es suficiente para disminuir la pobreza y que aquellos estados con condiciones de ocupar a la población en actividades manufactureras presentan condiciones más proclives a la disminución de la pobreza y la desigualdad, por lo que además se sugiere la relación entre la relocalización de los factores de la producción hacia sectores de mayor productividad y la disminución de la pobreza. 
Por otra parte, Padilla-Pérez y Villarreal (2017) estudian la relación entre el cambio en la estructura económica y el aumento de la productividad en el periodo de 1990 a 2015 en la economía mexicana; para esto descomponen el aumento en la productividad entre el efecto intrasectorial (el crecimiento que se atribuye a la relocalización de factores en otros sectores) e intersectorial (del mismo sector). Además, utilizan método de regresión donde explica el crecimiento en la productividad por los factores trabajo calificado y capital intensivo en conocimiento, encuentran significativa la relocalización de las horas trabajadas en la industria, pero su impacto se ve obstaculizado por los flujos de trabajo de sectores con alta productividad hacia aquellos con baja productividad (o a la baja).

En otro estudio, Micheli (2019), encuentra que en doce estados donde la industria automotriz ha tenido desarrollo se ha propiciado con esto un incremento en actividades manufactureras no automotrices y servicios avanzados (con alto grado de calificación), sin embargo, prevalece un contexto de mala calidad en remuneraciones y empleos.

La revisión llevada a cabo no da cuenta de trabajos de investigación que presenten el estudio de esta relación para el caso mexicano, de tal modo que la presente investigación pretende llenar ese vacío de información. Por otra parte, se pretende además, que al indagar si existe un vínculo entre la estructura económica mexicana y los niveles de pobreza, con ello sea posible aportar elementos que contribuyan al combate de esta.

\section{Metodología aplicada y datos utilizados}

Los estudios recientes sobre el cambio en la estructura económica y los procesos de industrialización en países en vías de desarrollo destacan la medición por medio del efecto reasignación sectorial o simplemente efecto reasignación (ER), el cual se define como la contribución a la variación en la productividad laboral de la movilización de trabajadores entre los diferentes sectores de un periodo con respecto a otro, siguiendo el trabajo de McMilllan y Rodrik (2011), ampliamente citado y el cual se expone a continuación:

$\Delta \mathrm{Yt}=\sum \theta \mathrm{i}, \mathrm{t}-\mathrm{k} \cdot \Delta \mathrm{yi}, \mathrm{t}+\sum \mathrm{yi}, \mathrm{t} \cdot \Delta \theta \mathrm{i}, \mathrm{t}$

Donde:

$" \Delta$ " se refiere al incremento en términos porcentuales de la variable en el periodo.

"Y" representa a la productividad entendida como la división del PIB de cada tipo de actividad en un año entre el número de personas ocupadas en la misma.

" $\theta$ " representa la participación de la población ocupada en el sector "i" en el año "t" de la ocupación total.

"i" representa la actividad económica, mientras que "t" hace referencia al año dentro del periodo.

" $\sum \theta \mathrm{i}, \mathrm{t}-\mathrm{k} \Delta \mathrm{Y} \mathrm{i}, \mathrm{t}$ " representaría el incremento natural de la productividad dentro de esa actividad, también llamado efecto intrasectorial.

El segundo componente " $\sum y i, t \cdot \Delta \theta \mathrm{i}, \mathrm{t}$ " es llamado efecto reasignación (ER), Si RE $>0$ significa que el empleo se ha relocalizado desde actividades de menor productividad hacia las de mayor productividad, contrariamente si $\mathrm{RE} \leq 0$, este efecto está contribuyendo reducir la productividad laboral. De esta manera, el cálculo del efecto reasignación da cuenta de que tanto contribuye el cambio de la estructura laboral al incremento de la productividad general en un periodo determinado. 
Para el cálculo de la ecuación 1 se entiende como productividad laboral el incremento del PIB a precios constantes de cada actividad o sector por persona ocupada en cada estado o región en un año con respecto al anterior (OIT, 2015). Ambos datos son proporcionados por el Instituto Nacional de Geografía y Estadística, el PIB en el apartado de cuentas nacionales a precios de 2013, mientras que para la población empleada se considera a la Población Ocupada en el cuarto trimestre de la Encuesta Nacional de Ocupación y Empleo para el periodo que va del año 2006 a 2018.

Para la medición de la pobreza se toma el índice de pobreza por ingreso y pobreza extrema por ingreso estimados respectivamente por el Consejo Nacional de Evaluación de la Política Social (CONEVAL). Estos son presentados cada dos años y son calculados en base a la Encuesta Nacional de Ingreso y Gasto de los Hogares (ENIGH) realizada bianualmente por el Instituto Nacional de Estadística y Geografía (INEGI).

El CONEVAL utiliza los índices de precios del Índice Nacional de Precios al Consumidor (INPC) para medir las Líneas de Pobreza por Ingresos (LP). Estas líneas se construyen midiendo el ingreso mensual mínimo para satisfacer las necesidades básicas nacionales (en zona urbana o rural) a partir de una canasta alimentaria para las líneas de pobreza extrema (LPE) y una no alimentaria que al sumarse a la anterior constituye la $\mathrm{LP}^{3}$. De tal manera que la pobreza por ingresos se calcula determinando el número de personas que perciben un ingreso mensual por debajo de la LP y la LPE dividiendo el de cada hogar entre el número de personas que lo habiten de acuerdo con la ENIGH (CONEVAL, 2020).

Por último, se estima un modelo de regresión lineal robusta considerando 192 observaciones en conjunto de datos de panel para explicar los niveles de pobreza moderada y extrema a partir de la estructura económica siguiendo la propuesta de Aggarwal y Kumar (2012) como se muestra en las ecuaciones 2 y 3 donde se explican ambos índices de pobreza por la participación en el PIB de los sectores secundario y terciario; el crecimiento económico y el efecto reasignación. También se utiliza la inversión en programas sociales como variable de control, ya que teórica y empíricamente se reconoce su contribución en la disminución de la pobreza (Aggarwal y Kumar, 2012; Lavopa y Szirmai 2012; Tello, 2015):

$$
\begin{aligned}
& \mathrm{PM}=\beta_{0}+\beta_{1} \mathrm{CE} i, \mathrm{t}+\beta_{2} \mathrm{PIB} 2_{\mathrm{i}, \mathrm{t}}+\beta_{3} \mathrm{PIB}_{\mathrm{i}, \mathrm{t}}+\beta_{4} \mathrm{ER}_{\mathrm{i}, \mathrm{t}}+\beta_{5} \mathrm{PSS}_{\mathrm{i}, \mathrm{t}}+\mathrm{Ei}_{\mathrm{i}} \quad \text { (Ecuación 2) } \\
& \mathrm{PE}=\beta_{0}+\beta_{1} \mathrm{CE} \mathrm{i}, \mathrm{t}+\beta_{2} \mathrm{PIB} 2 \mathrm{i}, \mathrm{t}+\beta_{3} \mathrm{PIB} 3 \mathrm{i}, \mathrm{t}+\beta_{4} \mathrm{ER}_{\mathrm{i}, \mathrm{t}}+\beta_{5} \mathrm{PS}_{\mathrm{i}, \mathrm{t}}+\mathrm{Ei} \quad \text { (Ecuación 3) } \\
& \mathrm{i}=1, \ldots 32 \mathrm{t}=1, \ldots 6
\end{aligned}
$$

Donde:

PM: representa el índice de pobreza moderada por ingresos presentado por el CONEVAL.

$P E$ : significa el índice de pobreza extrema por ingresos presentado por el CONEVAL.

$\beta 0$ :representa la constante.

$C E$ : representa el crecimiento económico registrado en el año "t" con respecto al anterior en el estado "i".

PIB2: representa la participación porcentual del sector secundario en el PIB.

PIB3: significa la participación del sector terciario en el PIB.

\footnotetext{
${ }^{3}$ En 2008 la LP urbana es de $\$ 2,009.13 \mathrm{MXN}$ mientras que la rural es de $\$ 1,266.69 \mathrm{MXN}$, en este mismo año la LPE urbana es de $\$ 921.47 \mathrm{MXN}$ y la LPE rural es de $\$ 653.78$ MXN. Para el año 2018 la LP urbana tiene un valor de $\$ 3,089.37 \mathrm{MXN}$, mientras que la rural es de $\$ 2,008.71 \mathrm{MXN}$, y la LPE es de $\$ 1,556.24 \mathrm{MXN}$ para las zonas urbana y de $\$ 1,113.23 \mathrm{MXN}$ para las rurales.
} 
$R E$ : representa el efecto reasignación calculado para cada estado/región en cada año calculado con la ecuación 1.

PS: Representa la inversión en programas sociales como porcentaje del PIB en cada estado y año.

$\mathcal{E}$ : representa el error estadístico.

Para las variables explicativas se consideran los años que coinciden con el índice de pobreza (2008, 2010, 2012, 2014 y 2016), respecto a las variaciones son considerados los cambios bianuales por la misma razón entre los mismos años. El crecimiento económico se considera como el incremento relativo del PIB registrado el año "i" con respecto al registrado dos años antes; la participación de los sectores en la economía se refiere al porcentaje que ocupa cada PIB (primario, secundario y terciario) en cada año y estado; como inversión en infraestructura (INF) se considera el porcentaje del PIB en cada estado y año, como proxy de la variable se toma la inversión de programas sociales (PS) se considera el monto destinado a "transferencias y apoyos" como porcentaje del PIB en cada año. Los datos son proporcionados por el Instituto Nacional de Geografía y Estadística (INEGI). Para el manejo de los datos se utiliza el software Excel y para la aplicación de los modelos estadísticos el software STATA 15.1.

\section{La estructura económica y pobreza en las regiones de México}

Antes de proceder al análisis descriptivo de la estructura económica y las condiciones de pobreza en México y sus regiones durante el periodo 2008-2018, se debe precisar que para facilitar dicho análisis se toma en cuenta la regionalización elaborada por el Consejo Nacional de Población (Conapo, 2004), la cual comprende cuatro grandes regiones compuestas por entidades federativas caracterizadas por su cercanía geográfica e intensidad migratoria (Mapa 1).

Estas regiones son: La región centro, que agrupa las entidades de Hidalgo, Estado de México, Morelos, Puebla, Tlaxcala, Querétaro y la Ciudad de México; La región norte, compuesta por los estados de Baja California, Baja California Sur, Chihuahua, Coahuila, Nuevo León, Sinaloa, Sonora y Tamaulipas; región sur-sureste que incluye a Campeche, Chiapas, Guerrero, Oaxaca, Quintana Roo, Tabasco, Veracruz y Yucatán; y la región tradicional comprendida por Aguascalientes, Colima, Durango, Guanajuato, Jalisco, Michoacán, Nayarit, San Luis Potosí y Zacatecas. 


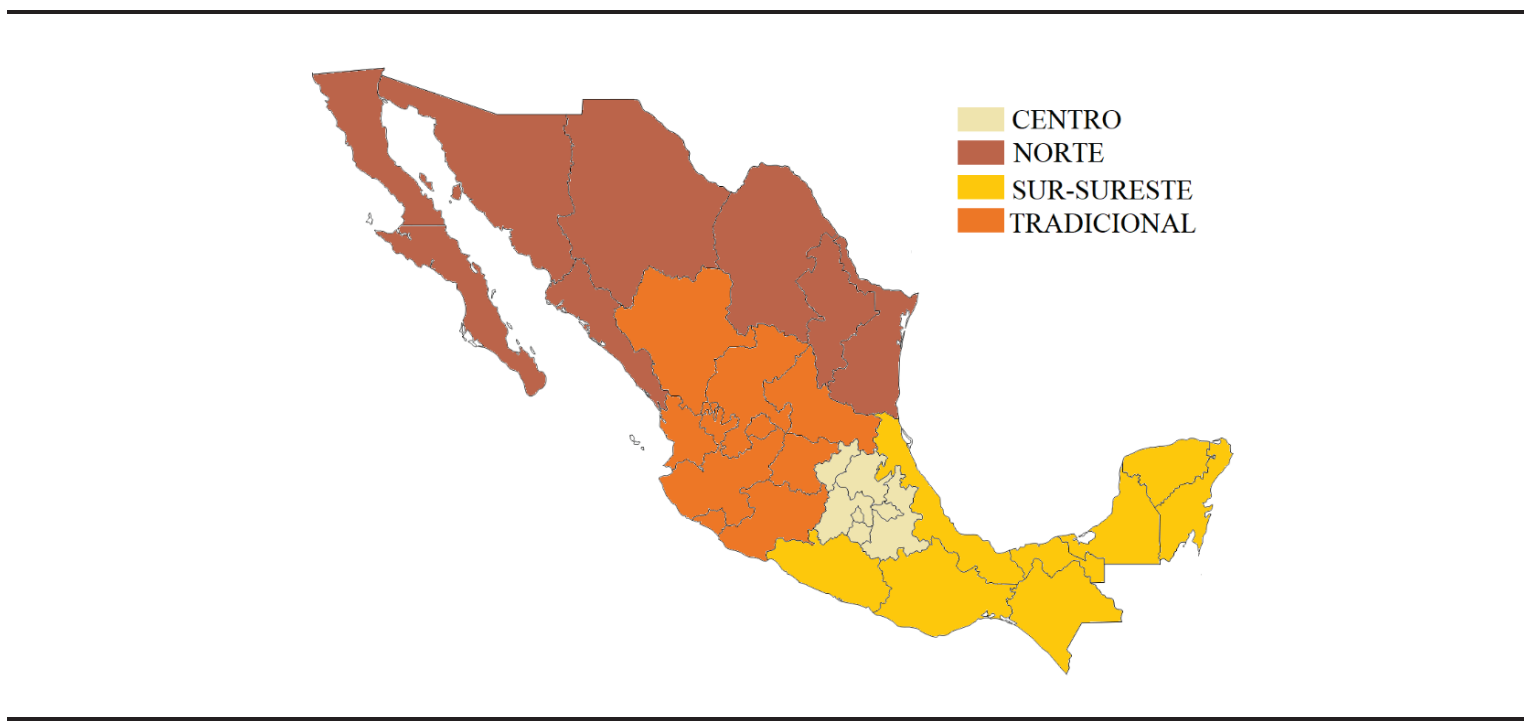

Fuente: Elaboración propia en base a la regionalización de CONAPO (2004)

A nivel nacional, el Cuadro 1 muestra que la estructura económica tiende a la terciarización, en mayor medida al considerar la participación sectorial del PIB y en menor medida si se toma en cuenta la ocupación laboral relativa en cada sector en el periodo de análisis 2008-2018 ${ }^{4}$. La participación del sector secundario se reduce en el PIB, pero sigue siendo el sector más importante después el terciario, tal relevancia se confirma al considerar la estructura del empleo pues el sector secundario provee la cuarta parte del empleo en el país. Por último, destaca el sector primario con una participación de apenas el 3.3\% en cuanto al PIB nacional, aunque dicho sector participa en promedio con el 13\% de la ocupación nacional.

\footnotetext{
${ }^{4}$ Las cifras con los que se trabaja a continuación son proporcionadas por el Instituto Nacional de Geografía y Estadística (INEGI), lo que se refiera a cuentas nacionales es encontrado en el Banco de Información Económica (BIE), estas son presentadas en millones de pesos base 2013.
} 
Cuadro 1

Estructura económica en México y sus regiones

\begin{tabular}{|c|c|c|c|c|c|c|c|c|c|c|c|c|}
\hline & \multicolumn{4}{|c|}{ Sector I } & \multicolumn{4}{|c|}{ Sector II } & \multicolumn{4}{|c|}{ Sector III } \\
\hline & \multicolumn{2}{|c|}{$P I B^{*}$} & \multicolumn{2}{|c|}{$P O^{* *}$} & \multicolumn{2}{|c|}{$P I B^{*}$} & \multicolumn{2}{|c|}{$P O^{* *}$} & \multicolumn{2}{|c|}{$P I B^{*}$} & \multicolumn{2}{|c|}{$P O^{* *}$} \\
\hline & 2008 & 2018 & 2008 & 2018 & 2008 & 2018 & 2008 & 2018 & 2008 & 2018 & 2008 & 2018 \\
\hline Total nacional & $3.35 \%$ & $3.34 \%$ & $14.14 \%$ & $12.69 \%$ & $36.51 \%$ & $30.55 \%$ & $25.79 \%$ & $25.58 \%$ & $60.14 \%$ & $66.11 \%$ & $59.34 \%$ & $61.21 \%$ \\
\hline Región centro & $1.43 \%$ & $1.21 \%$ & $8.56 \%$ & $7.69 \%$ & $23.22 \%$ & $20.09 \%$ & $26.16 \%$ & $24.96 \%$ & $75.35 \%$ & $78.70 \%$ & $64.91 \%$ & $67.06 \%$ \\
\hline Región norte & $3.81 \%$ & $3.74 \%$ & $8.32 \%$ & $6.95 \%$ & $41.38 \%$ & $38.27 \%$ & $29.96 \%$ & $31.16 \%$ & $54.80 \%$ & $57.99 \%$ & $59.54 \%$ & $60.51 \%$ \\
\hline Región sur-sureste & $3.46 \%$ & $3.76 \%$ & $26.92 \%$ & $25.29 \%$ & $55.07 \%$ & $39.76 \%$ & $19.87 \%$ & $18.46 \%$ & $41.48 \%$ & $56.48 \%$ & $52.94 \%$ & $56.04 \%$ \\
\hline Región tradicional & $6.39 \%$ & $6.21 \%$ & $15.44 \%$ & $13.64 \%$ & $31.41 \%$ & $30.69 \%$ & $27.27 \%$ & $27.83 \%$ & $62.20 \%$ & $63.10 \%$ & $56.91 \%$ & $58.20 \%$ \\
\hline
\end{tabular}

* Porcentaje de participación del sector en el PIB. ${ }^{* *}$ Porcentaje de participación del sector en la población ocupada.

Fuente: Elaboración propia con datos del Sistema de Cuentas Nacionales del INEGI a pesos del 2013 y la ENOE.

La jerarquía observada en el país en términos de la participación sectorial sea en cuanto al PIB o en la estructura del empleo, se replica en todas las regiones. De tal manera, destaca la región centro cuyo sector servicios participaba con el 78.7\% de su PIB en el 2018, seguida por las regiones tradicional (63.1\%), norte (57.9\%) y sur-sureste (56.4\%). Igual tiene preeminencia la región centro (67.0\%) al observar la participación del sector terciario en la ocupación de las regiones, seguida por la norte (60.5\%), tradicional (58.2\%) y sur-sureste $(56.0 \%)$.

En el sector secundario, respecto a la participación en el PIB sobresale la región sur-sureste (39.7\%) lo cual denota la relevancia de Pemex en la región, luego está la región norte con el 38.2\% de su PIB generado por el sector secundario, la región tradicional (30.6\%) y la centro (20.0\%), resultados que dan cuenta de un proceso de desconcentración industrial en el país. La estructura del empleo confirma la jerarquía industrial en las regiones, destacando la región norte con el 31.1\% de su ocupación en este sector para el año 2018, seguida por la región tradicional (27.8\%), la centro (24.9\%) y sur-sureste (18.4\%).

En cuanto al sector primario, al igual que el país las regiones observan la menor participación en términos de su PIB y ocupación; sin embargo, sobresale la región tradicional con el 6.2\% de su PIB generado en este sector para el 2018, le siguen las regiones norte y sur-sureste con el 3.7\% y la centro con el 1.21\% de su PIB. Al tomar en cuenta la composición sectorial del empleo, destaca la región sur-sureste con el 25.2\% de su ocupación en dicho sector en el año 2018, seguida por las regiones tradicional (13.6\%), centro (7.6\%) y norte (6.9\%).

En el Cuadro 2, se observa que el ER en todas las regiones presenta coeficiente positivo. Lo cual se explica a partir de que, si bien se atraviesa un proceso de desindustrialización durante el periodo, ya que las actividades a las que se reubica el empleo en todas las regiones son en su mayoría actividades de servicios y no industriales -como se aprecia en el Cuadro 1-; el empleo se relocaliza desde actividades agrícolas y de servicios (de baja productividad) a servicios con productividad laboral más elevada en todas las regiones (ya que el ER tiene coeficiente positivo) por excepción de la región sur-sureste donde el coeficiente ER es negativo. 
En el país el aumento en la productividad se atribuye principalmente al ER. En la región norte la productividad se atribuye también principalmente al ER y en la región tradicional cuatro quintas partes del incremento en la productividad se debe al EI y el resto al ER; sin embargo, en la región centro ambos efectos guardan el mismo peso. Por último, en la sur-sureste la reducción en la productividad se debe principalmente al EI y en menor medida al ER.

Cuadro 2 El efecto reasignación en México y sus regiones

\begin{tabular}{|c|c|c|c|c|c|c|c|c|}
\hline $2008-2018$ & & $\Delta Y t^{*}$ & & $E I^{\star *}$ & & $Q^{* \star *}$ & $E I \%$ & $E R \%$ \\
\hline Total nacional & $\$$ & 10,293 & $\$$ & 933 & $\$$ & 9,359 & $9 \%$ & $91 \%$ \\
\hline Región centro & $\$$ & 35,573 & $\$$ & 17,652 & $\$$ & 17,922 & $50 \%$ & $50 \%$ \\
\hline Región norte & $\$$ & 11,071 & $-\$$ & 266 & $\$$ & 11,338 & $-2 \%$ & $102 \%$ \\
\hline Región sur - sureste & $-\$$ & 62,091 & $-\$$ & 38,251 & $-\$$ & 23,840 & $62 \%$ & $38 \%$ \\
\hline Región tradicional & $\$$ & 33,819 & $\$$ & 24,538 & $\$$ & 9,280 & $73 \%$ & $27 \%$ \\
\hline
\end{tabular}

*Variación en la productividad. ${ }^{* *}$ Efecto Intrasectorial. ${ }^{* *}$ Efecto Reasignación.

Fuente: Elaboración propia en base al Sistema de Cuentas Nacionales del INEGI y la ENOE.

Es importante señalar que los índices de pobreza y pobreza extrema regional resultan del promedio de los índices obtenidos por el Coneval para los estados que conforman cada región. En el Cuadro 3, en términos de la pobreza moderada se puede ver que la región sur-sureste registra los índices más altos con un 68.1\% en el 2018, seguida por la región centro (52.4\%) y tradicional (47.2\%), mientras que, la región norte (35.6\%) tiene los índices de pobreza más bajos. Además, el mayor índice de pobreza extrema se aprecia en la región sur-sureste (34.4\%) para el 2018, seguida por la centro (14.6\%), tradicional (14.0\%) y norte (8.1\%). 
Niveles de pobreza en México y sus regiones

\begin{tabular}{lcccccc}
\hline \multicolumn{1}{c}{ Año } & \multicolumn{3}{c}{ Pobreza moderada* } & \multicolumn{3}{c}{ Pobreza Extrema** } \\
Total nacional & 2008 & 2018 & $\Delta^{* * *}$ & 2008 & 2018 & $\Delta^{* * *}$ \\
Centro & $49.00 \%$ & $48.80 \%$ & $-0.20 \%$ & $16.80 \%$ & $16.80 \%$ & $0.00 \%$ \\
Norte & $48.40 \%$ & $52.40 \%$ & $4.00 \%$ & $13.59 \%$ & $14.60 \%$ & $1.01 \%$ \\
Sur - Sureste & $35.36 \%$ & $35.66 \%$ & $0.30 \%$ & $9.06 \%$ & $8.13 \%$ & $-0.93 \%$ \\
Tradicional & $61.20 \%$ & $68.19 \%$ & $6.99 \%$ & $29.30 \%$ & $34.46 \%$ & $5.16 \%$ \\
\hline
\end{tabular}

*Población con ingreso inferior a la línea de pobreza por ingreso.

***oblación con ingreso inferior a la línea de pobreza extrema por ingreso.

${ }^{* * *}$ Variación de un periodo con respecto al otro.

Fuente: CONEVAL con datos de la ENIGH.

En suma, se puede apreciar que el efecto reasignación es más elevado en las regiones donde la pobreza (moderada y extrema) incrementa en menor proporción, mientras que en la región sur-sureste donde la pobreza incrementa considerablemente. Por su parte, en la región tradicional la pobreza disminuye y el efecto reasignación no es superior al efecto intrasectorial, sin embargo, ambos coeficientes son positivos.

De tal manera, el incremento en la productividad deriva de la relocalización del empleo en la estructura económica de las diferentes regiones en México, así como en menor medida del incremento natural; por lo tanto, ambos efectos resultan trascendentes ya que la región más pobre (sur-sureste) y con mayor incremento en esta a la par atraviesa desindustrialización y pérdida de productividad durante el período 2008-2018.

\section{Resultados del modelo econométrico}

Se estima una regresión lineal robusta considerando 192 observaciones en conjunto de datos de panel (ya que combinan una dimensión temporal y transversal), para determinar que tanto son explicados los niveles de pobreza moderada y pobreza extrema por el crecimiento económico, la participación de los sectores secundario y terciario en PIB; el efecto reasignación y la inversión en programas sociales como porcentaje del PIB de cada estado. Debido a estar trabajando con datos que parten del PIB, además de la presencia de datos atípicos en el cálculo del efecto reasignación (ER) se hace uso de la regresión robusta, de esta manera se ajustan las limitaciones tradicionales de la estimación por mínimos cuadrados ordinarios: datos anómalos, falta de normalidad y simetría en los errores (Andersen, 2008), de modo que no resulta necesario aplicar prueba de heteroscedasticidad.

Los resultados del modelo en relación con la pobreza moderada (Ecuación 2) denota alto poder explicativo con un $\mathrm{R}^{2}$ de $37 \%$; mientras que, la probabilidad del estadístico " $\mathrm{F}$ " es menor al 5\%, lo que indica que en conjunto estas variables pueden explicar los niveles de pobreza moderada. Además, la regresión estimada muestra que todas las variables observan un coeficiente con el signo esperado (excepto el gasto social) y en términos estadísticos resultaron significativas el PIB secundario, terciario y el gasto social (pero no el efecto reasignación ni el crecimiento económico), pues la probabilidad arrojada por el estadístico "t" es menor al 5\%. El hecho de que el signo del Efecto Reasignación sea el esperado, pero estadísticamente no significativo, quizá sea reflejo de que los incrementos en productividad no necesariamente se sustentan en un cambio estructural. 


\begin{tabular}{|c|c|c|c|c|}
\hline \multirow{2}{*}{$\begin{array}{l}\text { Prob }>F= \\
R- \\
\text { squared }=\end{array}$} & \multicolumn{2}{|c|}{$\begin{array}{c}P M \\
0\end{array}$} & \multicolumn{2}{|c|}{$\begin{array}{c}P E \\
0\end{array}$} \\
\hline & Coef. & $P>t$ & Coef. & $P>t$ \\
\hline CE & -0.343925 & 0.197 & -0.294333 & 0.088 \\
\hline PIB2 & -0.712271 & 0.013 & -0.418239 & 0.042 \\
\hline PIB3 & -0.941943 & 0.001 & -0.595668 & 0.004 \\
\hline ER & -0.000155 & 0.158 & -0.000107 & 0.142 \\
\hline PS & 2.165849 & 0 & 1.976543 & 0 \\
\hline _cons & 1.195724 & 0 & 0.5770818 & 0.004 \\
\hline
\end{tabular}

$\mathrm{Al}$ correr el modelo respecto a la pobreza extrema (Ecuación 3) se incrementa el poder explicativo, ya que registra un $\mathrm{R}^{2}$ de $45 \%$; mientras que, la probabilidad del estadístico " $F$ " es menor al $5 \%$, lo que indica que en conjunto estas variables explican los niveles de pobreza extrema. Nuevamente todas las variables tienen un coeficiente con el signo esperado (excepto el gasto social) y también resultaron significativas en términos estadísticos ya que la probabilidad del estadístico "t” es menor al 5\% (excepto el ER).

En resumen, los resultados del modelo econométrico indican que el CE, PIB2, PIB3 y ER reducen la pobreza, pero el ER es no significativo, es decir que elevados niveles de relocalización no se traducen en bajos niveles de pobreza debido a que los estados (principalmente los que integran la región sur-sureste) que ostentan altos niveles no incrementan la productividad natural de sus actividades y aunque la relocalización sea positiva no quiere decir que el trabajo migre hacia las actividades de mayor productividad en general.

Los resultados sugieren que elevados niveles de participación de los sectores secundario y terciario resultan en niveles de pobreza por debajo de la media nacional, lo cual es explicado a partir de que los estados que la padecen en menor medida se identifican con vocación manufacturera con elevada participación del sector secundario (los estados integrantes de las regiones norte y centro y en la región tradicional: Aguascalientes, Guanajuato y Jalisco).

\section{Conclusiones}

Se estudia la estructura económica en las regiones de México y si los cambios que esta experimenta se correlacionan e inciden en la pobreza regional durante el periodo 2008-2018. Para ello se calcula la participación relativa sectorial en el PIB y el empleo, el efecto intrasectorial (EI), el efecto de reasignación (ER) y se recurre a los índices de pobreza estimado por el Coneval. También se estima un modelo econométrico para identificar los factores determinantes de la pobreza. 
Se encuentra que la región norte destaca por niveles de pobreza muy por debajo de la media nacional y una estructura económica orientada a la industria con respecto a la misma. En las regiones centro y tradicional los niveles de pobreza son cercanos a la media nacional y su estructura ecnómica similar en la participación de los sectores. Por su parte, la región sur-sureste cuenta con una estructura principalmente agrícola y de baja ocupación industrial, además de encontrar en esta región elevados niveles de pobreza.

Se constata que la estructura económica tiende a terciarizarse en las regiones y el país, más aún tomando en cuenta la participación sectorial en el PIB y en menor medida al considerar la participación del empleo. La región centro destaca con la mayor participación del sector servicios (78.7\%) en su PIB y de igual manera tiene preeminencia al considerar la estructura del empleo (67.0\%). En consecuencia, jerarquía de la región centro en el sector secundario se diluye en el PIB (20\%) y el empleo (24.9\%) durante la década, lo que sugiere quizá un proceso de desconcentración industrial.

El efecto reasignación (ER) ilustra que el aumento de la productividad le es atribuible en las regiones de estudio con excepción de la región sur-sureste donde este coeficiente es negativo. Por su parte, el incremento en la productividad es inducido en mayor medida por la relocalización laboral, que por el incremento natural de esta tanto en las regiones norte y centro como en el país.

El aumento de la productividad es atribuible al efecto de reasignación (ER) en las diferentes regiones, este incremento es inducido en mayor medida por la relocalización laboral hacia empleos de mayor productividad, que por el incremento natural de la productividad en las regiones norte y centro, como también en el país.

Esto se explica por el hecho de que la participación del empleo en el sector industrial (el de mayor productividad) no se reduce, mientras que el empleo disminuye en las actividades agrícolas donde se encuentra la menor productividad y aumenta considerablemente en las actividades del sector terciario. No obstante, el empleo no se reubica en el sector de mayor productividad.

Por otra parte, se aprecia que el efecto reasignación es más elevado en regiones donde los niveles de pobreza son menos elevados, mientras que en la región sur-sureste (región con altos niveles de pobreza) la reasignación disminuye la productividad; sin embargo, en la región tradicional la pobreza disminuye y el efecto reasignación no es superior al efecto intrasectorial, pero ambos son positivos.

Por lo tanto, el incremento en la productividad deriva de la relocalización del empleo en la estructura económica de las diferentes regiones y, en menor medida del incremento natural. Aunque ambos efectos resultan relevantes, ya que la región más pobre (sur-sureste) y con mayor incremento en esta, a la vez atraviesa desindustrialización y pérdida de productividad durante el período 2008-2018.

Por último, según los modelos econométricos se encontró que la reducción en los niveles de la pobreza tanto moderada como extrema- se explica por el crecimiento económico, la participación de los sectores secundario y terciario en la economía, pero no por el efecto de reasignación, lo cual denota la inexistencia de un cambio estructural en las economías regionales y nacional.

Estos resultados, derivan en las siguientes recomendaciones: $1^{\circ}$, el Estado debe impulsar programas y políticas orientadas al desarrollo tecnológico masificando la educación superior y protegiendo a las empresas paraestatales estratégicas de acuerdo con las condiciones de cada región; $2^{\circ}$, considerando la heterogeneidad estructural entre las regiones del país, es fundamental priorizar el desarrollo en aquellas con altos niveles de pobreza, promoviendo la ubicación de actividades industriales y mecanismos de vinculación con el resto del país, particularmente en la región sur. 


\section{Referencias Bibliográficas}

Adair, C. (2006). Structural Change, Inequality, and Growth in Mexico. The University of Texas Inequality Project (Vol. 35).

Aggarwal, A., y Kumar, N. (2012). Structural Change, Industrialization and Poverty Reduction: The Case of India. South and South-West Asia Development. Papers No. 35. ESCAP Development Paper. New Delhi.

Andersen, R. (2008). Modern Methods for Robust Regression. Sage University Paper Series on Quantitative Applications in the Social Sciences, 07-152.

Berthélemy, J. (2018). Growth Acceleration, Structural Change, and Poverty Reduction in Africa and Poverty Reduction in Africa. African Economic Outlook 2018.

Calderón-Villarreal, C., \& Hernández-Bielma, L. (2016). Cambio estructural y desindustrialización en México. Panorama Económico, 12(23), 29-54.

Camberos C. M., y Bracamontes, N. J. (2015). Las crisis económicas y sus efectos en el mercado de trabajo, en la desigualdad y en la pobreza de México. Contaduría y administración, 60, 219-249.

Campos, R. M., y Luis, V. (2016). La relación entre crecimiento económico y pobreza en México. Investigación Económica, 75(298), 77-113.

Capdevielle. (2005). Globalización, especialización y heterogeneidad estructural en México. En M. Cimoli (Ed.), Heterogeneidad estructural, asimetrías tecnológicas y crecimiento en América Latina (pp. 9-39). Santiago: CEPAL.

Carrillo Arteaga, B. E., y Cadena Vargas, E. (2019). Terciarización y configuración espacial en México. Acta Universitaria, 29.

Economic Commission for Latin America and the Caribbean, ECLAC (2012). Cambio estructural para la igualdad: una visión integrada del desarrollo. Santiago.

- (2007) Los objetivos de desarrollo del Milenio y los desafíos para América Latina y el Caribe para avanzar en mayor bienestar, mejor capital humano y más igualdad de oportunidades. Documento de proyecto. Santiago.

- (2016) Agenda 2030 y los Objetivos de Desarrollo Sostenible. Una oportunidad para América Latina y el Caribe. Santiago.

Cimoli, M. (2005). Cambio estructural, heterogeneidad productiva y tecnológica en América Latina. En M. Cimoli (Ed.), Heterogeneidad estructural, asimetrías tecnológicas y crecimiento en América Latina. 9-39. Santiago: CEPAL.

Cimoli, M., G. Dosi and J.E. Stiglitz (2009) Industrial Policy and Development. The Political Economy of Capabilities Accumulation. Oxford University Press.

Cimoli, M., G. Porcile y S. Rovira (2010). Structural change and the BOP-constraint: why did Latin America fail to converge? Cambridge Journal of Economics, vol. 34, No 2.

Consejo Nacional de Evaluación de la Política Social (2016). Evolución de las Dimensiones de la pobreza 19902014. Ciudad de México.

- (2019). 10 años de medición de la pobreza en México, avances y retos en política social. Comunicado de prensa No. 10. Ciudad de México.

- (2020). Actualización de las Líneas de Pobreza por Ingreso. Ciudad de México.

Diao, X., McMillan, M., \& Rodrik, D. (2019). The recent growth boom in developing economies: A structuralchange perspective. The Palgrave Handbook of Development Economics (pp. 281-334).

Dutt, A. K. (2019). Structuralists, Structures, and Economic Development. In the Palgrave Handbook of Development Economics (pp. 109-141).

García, J. (2002). Liberalización, cambio estructural y crecimiento económico en Colombia. Cuadernos de Economía, 21(36), 189-229.

Harvey, D. (2007). Breve historia del neoliberalismo (Vol. 49). Ediciones Akal.

Instituto Nacional de Estadística y Geografía (INEGI). 2018. Banco de Información Económica (BIE). México.

International Labor Organization. (2015). Indicadores Clave del Mercado de Trabajo 2015: Informe completo. Oficina Internacional del Trabajo. Ginebra. 
Iniguez-montiel, A. J., \& Kurosaki, T. (2015). Limits to Economic Growth and the Effect of Redistribution in Mexico. PRIMCED Discussion Paper Series, (63), 1-42.

Keesing, D. B. (1968). Structural Change Early in Development: Mexico's Changing Industrial and Occupational Structure from 1895 to 1950. The Journal of Econoic History, 29(4), 716-738.

Lanzarotti, M. (1991). Chocs exogenes, ajustement et changement structurel en Corée du Sud. Revue Tiers Monde, 32(127), 633-653.

Lavinas, L., y Simões, A. (2016). Social Policy and Structural Heterogeneity in Latin America: The Turning Point of the 21St Century. Revista de Economia Contemporânea, 21(2), 1-35.

Lavopa, A., y Szirmai, A. (2012). Industrialization, employment, and poverty. UNU-MERIT Working Papers.

McMillan, M. S. y Rodrik, D. (2011). Globalization, Structural Change and Productivity Growth. NBER Working Paper No. 17143. Cambridge.

McMillan, M., Rodrik, D., y Verduzco-Gallo. (2014). Globalization, Structural Change, and Productivity Growth, with an Update on Africa. World Development, 63, 11-32.

Micheli Thirion, J. (2019). Cambio estructural en los estados especializados en producción automotriz de México, 1998-2013. Región y Sociedad, 31, e1110.

Moncayo, E. (2008). Cambio estructural: trayectoria y vigencia de un concepto. Structural change: Path and Validity of a concept. Revista CIFE, 32(13), 235-249.

Morton, A. D. (2003). Structural change and neoliberalism in Mexico: "Passive revolution" in the global political economy. Third World Quarterly, 24(4), 631-653.

Osmani, S. R. (1990). Structural Change and Poverty in Bangladesh: The Case of a False Turning Point. The Bangladesh Development Studies, 18(3), 55-74.

Padilla-Pérez, R., y Villarreal, F. G. (2017). Structural change and productivity growth in Mexico, 1990-2014. Structural Change and Economic Dynamics, 41, 53-63.

Pasinetti, L. L. (1973). The notion of vertical integration in economic analysis. Metroeconomica, vol. 25, No 1. -(1981). Structural Change and Economic Growth. A Theoretical Essay on the Dynamics of the Wealth of Nations. Cambridge, Cambridge University Press..

Prebisch, R. (1949), El desarrollo de la América Latina y algunos de sus principales problemas, Santiago, Comisión Económica para América Latina y el Caribe (CEPAL). -(1967), Hacia una dinámica del desarrollo latinoamericano, vol. 2, Ediciones de la Banda Oriental.

Segovia A. (1998). Cambio estructural, políticas macroeconómicas y pobreza en El Salvador. Política macroeconómica y pobreza en América y el Caribe, 489-540. Santiago de Chile: CEPAL.

Silva E. G. and A.C. Teixeira, (2008). Surveying structural change: Seminal contributions and a bibliometric account. Structural Change and Economic Dynamics, 19(4): 273-300.

Storm, S. (2015). Structural change. Development and Change, 46(4), 666-699.

Tello, M. D. (2015). Poverty, growth, structural change and social inclusion programs: a regional analysis of Peru. Regional and Sectoral Economic Studies, 15(2), 59-78.

United Nations Organization for Industrial Development. (2012). Structural Change, Poverty Reduction and Industrial Policy in the BRICS, 1-179.

Verme, P. (2010). A Structural Analysis of Growth and Poverty in the Short-Term. The Journal of Developing Areas, 43(2), 19-39. Retrieved from.

World Bank, (2018). Según Banco Mundial, la pobreza extrema a nivel mundial continúa disminuyendo, aunque a un ritmo más lento. Comunicado de prensa del Banco Mundial. Washington.

Yoguel E. Y Barletta F. (2017). ¿De qué hablamos cuando hablamos de cambio estructural? Manufactura y cambio estructural: Aportes para pensar la política industrial en la Argentina (27-54). Santiago: CEPAL. 